\title{
Finite Element Analysis of Radiation and Mass Transfer Flow Past Semi- Infinite Moving Vertical Plate with Viscous Dissipation
}

\author{
V. S. Rao ${ }^{1 \dagger}$, L. A. Babu and R. S. Raju ${ }^{2}$ \\ ${ }^{I}$ Department of Mathematics, Anurag group of Institutions, Hyderabad-500088, India. \\ ${ }^{2}$ Department of Mathematics, B.V.R.I.T, Narsapur-502313, India.
}

†Corresponding Author Email: uhita@yahoo.com

(Received April 23, 2010; accepted July 21, 2012)

\begin{abstract}
The objectives of the present study are to investigate the radiation effects on unsteady heat and mass transfer flow of a chemically reacting fluid past a semi-infinite vertical plate with viscous dissipation. The method of solution is applied using Finite element technique. Numerical results for the velocity, the temperature and the concentration are shown graphically for various flow parameters. The expressions for the skin-frication, Nusselt number and Sherwood number are obtained. The result shows that increased cooling $(\mathrm{Gr}>0)$ of the plate and the Eckert number leads to a rise in the velocity. Also, an increase in the Eckert number leads to an increase in the temperature, whereas increase in radiation lead to a decrease in the temperature distribution when the plate is being cooled.
\end{abstract}

Keywords: Transfer, Viscous dissipation, Radiation, Chemical reaction, Finite element technique.

\section{NOMENCLATURE}

$C$ - Dimensional concentration

$c_{p}$ - Specific heat at constant pressure

$D$ - Mass diffusivity

$g$ - Acceleration due to gravity

$k_{r}^{\prime}$ - Chemical reaction parameter

$T$ - Dimensional temperature

$t^{\prime}$ - Time

\section{INTRODUCTION}

For some industrial applications such as glass production and furnace design in space technology applications, cosmial flight aerodynamics rocket, propulsion systems, plasma physics which operate at higher temperatures, radiation effects can be significant. Soundalgekar and Takhar (1993) considered the radiative free convection flow of an optically thin greygas past a semi-infinite vertical plate. Radiation effects on mixed convection along an isothermal vertical plate were studied by Hussian and Takhar (1996). Raptis and Perdikis (1999) have studied the effects of thermal radiation and free convection flow past a moving vertical plate.

\author{
$u^{\prime}, v^{\prime}$ - Velocity components in $x^{\prime}, y^{\prime}$ Directions \\ respectively \\ $\alpha$ - Fluid thermal diffusivity \\ $\beta$ and $\beta^{*}$ - Thermal and concentration expansion \\ Coefficient \\ $\mu$ - Coefficient of viscosity \\ $v$ - Kinematic viscosity \\ $\rho$ - The fluid density
}

Chamkha et al. (2001) analyzed the effects of radiation on free convection flow past a semi-infinite vertical plate with mass transfer. Kim and Fedorov (2003) studied transient mixed radiative convection flow of a micro polar fluid past a moving, semi-infinite vertical porous plate. Prakash and Ogulu (2006) have investigated an unsteady two-dimensional flow of a radiating and chemically reacting fluid with time dependent suction.

In many chemical engineering processes, there does occur the chemical reaction between a foreign mass and the fluid in which the plate is moving. These processes take place in numerous industrial applications viz., Polymer production, manufacturing of ceramics or glassware and food procession. Das et al. (1994) have 
studied the effects of mass transfer on flow past an impulsively started infinite vertical plate with constant heat flux and chemical reaction.

In all these investigations, the viscous dissipation is neglected. The viscous dissipation heat in the natural convective flow is important, when the flow field is of extreme size or at low temperature or in high gravitational field. Gebhar (1962) shown the importance of viscous dissipative heat in free convection flow in the case of isothermal and constant heat flux in the plate. Soundalgekar (1972) analyzed the effect of viscous dissipative heat on the two dimensional unsteady, free convective flow past an vertical porous plate when the temperature oscillates in time and there is constant suction at the plate. Israel Cookey et al [18] investigated the influence of viscous dissipation and radiation on unsteady MHD free convection flow past an infinite heated vertical plate in porous medium with time dependent suction.

The objective of the present paper is to analyze the radiation and mass transfer effects on an unsteady twodimensional laminar convective boundary layer flow of a viscous, incompressible, chemically reacting fluid along a semi-infinite vertical plate with suction, by taking into account the effects of viscous dissipation. The equations of continuity, linear momentum, energy and diffusion, which govern the flow field are solved by using finite element technique. The behavior of the velocity, temperature, concentration has been discussed for variations in the governing parameters.

\section{Mathematical ANALYSIS}

An unsteady two-dimensional laminar boundary layer flow of a viscous, incompressible, radiating fluid along a semi-infinite vertical plate in the presence of thermal and concentration buoyancy effects is considered, by taking the effect of viscous dissipation into account. The $x^{\prime}$-axis is taken along the vertical infinite plate in the upward direction and the $y^{\prime}$-axis normal to the plate. The level of concentration of foreign mass is assumed to be low, So that the Soret and Dufour effects are negligible. Now under Boussinesq's approximation, the flow field is governed by the following equations:

$\frac{\partial v^{\prime}}{\partial y^{\prime}}=0$

$\frac{\partial u^{\prime}}{\partial t^{\prime}}+v^{\prime} \frac{\partial u^{\prime}}{\partial y^{\prime}}=$

$v \frac{\partial^{2} u^{\prime}}{\partial y^{\prime 2}}+g \beta\left(T-T_{\infty}\right)+g \beta^{*}\left(C-C_{\infty}\right)-\frac{\sigma B_{o}^{2} u^{\prime}}{\rho}$

$\frac{\partial T}{\partial t^{\prime}}+v^{\prime} \frac{\partial T}{\partial y}=\alpha \frac{\partial^{2} T}{\partial y^{\prime 2}}-\frac{1}{\rho c_{p}} \frac{\partial q_{r}}{\partial y^{\prime}}+\frac{\mu}{\rho c_{p}}\left(\frac{\partial u^{\prime}}{\partial y^{\prime}}\right)^{2}$

$\frac{\partial C}{\partial t^{\prime}}+v^{\prime} \frac{\partial C}{\partial y^{\prime}}=D \frac{\partial^{2} C}{\partial y^{\prime 2}}-k_{r}^{\prime 2}\left(C-C_{\infty}\right)$

Where $u^{\prime}, v^{\prime}$ are the velocity components in $x^{\prime}, y^{\prime}$ directions respectively. $t^{\prime}-$ the time, $\rho$-the fluid density, $v$ - the kinematic viscosity, $c_{p}$ - the specific heat at constant pressure, $g$-the acceleration due to gravity, $\beta$ and $\beta^{*}$ - the thermal and concentration expansion coefficient respectively, $\boldsymbol{T}$ - the dimensional temperature, $C$ - the dimensional concentration, $\alpha$ the fluid thermal diffusivity, $\mu$ - coefficient of viscosity, $D$ - the mass diffusivity, $k_{r}^{\prime}$ - the chemical reaction parameter.

The boundary conditions for the velocity, temperature and concentration fields are

$$
\begin{aligned}
& u^{\prime}=U_{0}, T=T_{w}+\varepsilon\left(T_{w}-T_{\infty}\right) e^{n^{\prime} t^{\prime}}, \\
& C=C_{w}+\varepsilon\left(C_{w}-C_{\infty}\right) e^{n^{\prime} t^{\prime}} \quad \text { at } y^{\prime}=0 \\
& u^{\prime} \rightarrow 0, T \rightarrow T_{\infty}, T \rightarrow T_{\infty}, \text { as } y^{\prime} \rightarrow \infty
\end{aligned}
$$

Where $U_{0}$ is the scale of free stream velocity, $T_{w}$ and $C_{w}$ are the wall dimensional temperature and concentration respectively, $T_{\infty}$ and $C_{\infty}$ are the free stream dimensional temperature and concentration respectively, $n^{\prime}$ - the constant.

By using Rosseland approximation, the radiative heat flux is given by

$q_{r}=-\frac{4 \sigma_{s}}{3 K_{e}} \frac{\partial T^{4}}{\partial y^{\prime}}$

Where $\sigma_{s}$ - the Stefan-Boltzmann constant and $K_{e}$ the mean absorption coefficient. It should be noted that by using Rosseland approximation, the present analysis is limited to optically thick fluids. If temperature differences within the flow are sufficiently small, then Eq. (6) can be linearised by expanding $T^{4}$ in the Taylor series about $T_{\infty}$ and neglecting higher order terms, we obtain

$$
T^{4} \approx 4 T_{\infty}^{3} T-3 T_{\infty}^{4}
$$

In view of Eq. (6) and Eq. (7), Eq. (3) reduces to

$$
\begin{aligned}
& \frac{\partial T}{\partial t^{\prime}}+v^{\prime} \frac{\partial T}{\partial y^{\prime}}= \\
& \frac{k}{\rho c_{p}} \frac{\partial^{2} T}{\partial y^{\prime 2}}+\frac{16 \sigma_{s}}{3 \rho c_{p} K_{e}} T_{\infty}^{3} \frac{\partial^{2} T}{\partial y^{\prime 2}}+\frac{\mu}{\rho c_{p}}\left(\frac{\partial u^{\prime}}{\partial y^{\prime}}\right)^{2}
\end{aligned}
$$

From the continuity Eq. (1), it is clear that suction velocity normal to the plate is either a constant or function of time. Hence, it is assumed in the form

$v^{\prime}=-V_{0}\left(1+\varepsilon A e^{n^{\prime} t^{\prime}}\right)$

Where $\mathrm{A}$ is a real positive constant, $\varepsilon$ and $\varepsilon \mathrm{A}$ are small values less than unity and $\mathrm{VO}$ is scale of suction velocity at the plate surface. 
In order to write the governing equations and the boundary condition in dimension less form, the following non- dimensional quantities are introduced.

$$
\begin{aligned}
& u=\frac{u^{\prime}}{U_{0}}, \quad y=\frac{V_{0} y^{\prime}}{v}, t=\frac{V_{0}^{2} t^{\prime}}{v}, \quad n=\frac{v n^{\prime}}{V_{0}^{2}}, \\
& \theta=\frac{T-T_{\infty}}{T_{w}-T_{\infty}}, \varphi=\frac{C-C_{\infty}}{C_{w}-C_{\infty}}, \operatorname{Pr}=\frac{v \rho C_{p}}{k}=\frac{v}{\alpha}, \\
& S c=\frac{v}{D}, G r=\frac{g \beta v\left(T_{w}-T_{\infty}\right)}{U_{0} V_{0}^{2}}, \\
& G m=\frac{g \beta^{*} v\left(C_{w}-C_{\infty}\right)}{U_{0} V_{0}^{2}}, E c=\frac{U_{0}^{2}}{c_{p}\left(T_{w}-T_{\infty}\right)}, \\
& k_{r}^{2}=\frac{k_{r}^{\prime 2} v}{V_{0}^{2}}, R=\frac{16 \sigma_{s} T_{\infty}^{3}}{3 K_{e} k}, M=\frac{\sigma B_{o}^{2} v}{\rho V_{o}^{2}}
\end{aligned}
$$

In view of the Eqs. (6) - (10), Eqs. (2) - (4) reduce to the following dimensionless form.

$$
\frac{\partial u}{\partial t}-\left(1+\varepsilon A e^{n t}\right) \frac{\partial u}{\partial y}=\frac{\partial^{2} u}{\partial y^{2}}+G r \theta+G m \varphi-M^{2} u
$$

$$
\frac{\partial \theta}{\partial t}-\left(1+\varepsilon A e^{n t}\right) \frac{\partial \theta}{\partial y}=\left(\frac{1+R}{\operatorname{Pr}}\right) \frac{\partial^{2} \theta}{\partial y^{2}}+E c\left(\frac{\partial u}{\partial y}\right)^{2}
$$

$\frac{\partial \varphi}{\partial t}-\left(1+\varepsilon A e^{n t}\right) \frac{\partial \varphi}{\partial y}=\frac{1}{S c} \frac{\partial^{2} \varphi}{\partial y^{2}}-k_{r}^{2} \varphi$

where $G r, G m, \operatorname{Pr}, R, E c, S c \quad$ and $\quad k_{r}$ are the thermal Grashof number , Modified Grashof number, Prandtl number, Radiation parameter, Eckert number, Schmidt number and Chemical reaction parameter respectively.

The corresponding boundary conditions are

$$
\begin{array}{ll}
u=0.5, \theta=1+\varepsilon e^{n t}, \varphi=1+\varepsilon e^{n t} & \text { at } \mathrm{y}=0 \\
u \rightarrow 0, \theta \rightarrow 0, \varphi \rightarrow 0 & \text { as } y \rightarrow \infty
\end{array}
$$

\section{Solution Of The Problem}

The Galerkin equation for the differential Eq. (11) becomes

$$
\int_{y_{j}}^{y_{k}} N^{(e)^{T}}\left[\frac{\partial^{2} u^{(e)}}{\partial y^{2}}+P \frac{\partial u^{(e)}}{\partial y}-\frac{\partial u^{(e)}}{\partial t}-M^{2} u^{(e)}+R\right] d y=0
$$

Where $P=1+\varepsilon A e^{n t}, R=G r \theta+G m \varphi$.

Let the linear piecewise approximation solution
$u^{(e)}=N_{j}(y) u_{j}(t)+N_{k}(y) u_{k}(t)=N_{j} u_{j}+N_{k} u_{k}$

where $N_{j}=\frac{y_{k}-y}{y_{k}-y_{j}}, N_{k}=\frac{y-y_{j}}{y_{k}-y_{j}}$

$\left.N^{(e)^{T}} \frac{\partial u^{(e)}}{\partial y}\right\}_{y_{j}}^{y_{k}}-$

$\int_{y_{j}}^{y_{k}}\left\{\begin{array}{l}\frac{\partial N^{(e)^{T}}}{\partial y} \frac{\partial u^{(e)}}{\partial y}- \\ N^{(e)^{T}}\left(P \frac{\partial u^{(e)}}{\partial y}+\frac{\partial u^{(e)}}{\partial t}-M^{2} u^{(e)}-R\right)\end{array}\right\} d y=0$

Neglecting the first term in Eq. (16) we get

$\int_{y_{j}}^{y_{k}}\left\{\frac{\partial N^{(e)^{T}}}{\partial y} \frac{\partial u^{(e)}}{\partial y}-N^{(e)^{T}}\left(P \frac{\partial u^{(e)}}{\partial y}-\frac{\partial u^{(e)}}{\partial t}-M^{2} u^{(e)}+R\right)\right\} d y=0$

After integrating term by term in the interval yj to $\mathrm{yk}$

$\frac{1}{l^{(e)}}\left[\begin{array}{cc}1 & -1 \\ -1 & 1\end{array}\right]\left[\begin{array}{l}u_{j} \\ u_{k}\end{array}\right]-\frac{P}{2}\left[\begin{array}{ll}-1 & 1 \\ -1 & 1\end{array}\right]\left[\begin{array}{l}u_{j} \\ u_{k}\end{array}\right]+\frac{l^{(e)}}{6}\left[\begin{array}{ll}2 & 1 \\ 1 & 2\end{array}\right]\left[\begin{array}{c}u_{j}^{\bullet} \\ u_{k}^{\bullet}\end{array}\right]$

$+\frac{M^{2} l}{6}\left[\begin{array}{ll}2 & 1 \\ 1 & 2\end{array}\right]\left[\begin{array}{l}u_{j} \\ u_{k}\end{array}\right]=R \frac{l^{(e)}}{2}\left[\begin{array}{l}1 \\ 1\end{array}\right]$

Where $l^{(e)}=y_{k}-y_{j}=h$ and $\operatorname{dot}$ denotes the differentiation with respect to $t$.

We write the element equations for the elements $y_{i-1} \leq y \leq y_{i}$ and $y_{i} \leq y \leq y_{i+1_{i}}$ assemble three element equations, we obtain

$\frac{1}{l^{(e)}}\left[\begin{array}{ccc}1 & -1 & 0 \\ -1 & 2 & -1 \\ 0 & -1 & 1\end{array}\right]\left[\begin{array}{c}u_{i-1} \\ u_{i} \\ u_{i+1}\end{array}\right]-\frac{P}{2}\left[\begin{array}{ccc}-1 & 1 & 0 \\ -1 & 0 & 1 \\ 0 & -1 & 1\end{array}\right]\left[\begin{array}{c}u_{i-1} \\ u_{i} \\ u_{i+1}\end{array}\right]$

$+\frac{M^{2} l}{6}\left[\begin{array}{ccc}2 & 1 & 0 \\ 1 & 4 & 1 \\ 0 & 1 & 2\end{array}\right]\left[\begin{array}{c}u_{i-1} \\ u_{i} \\ u_{i+1}\end{array}\right]+\frac{l^{(e)}}{6}\left[\begin{array}{ccc}2 & 1 & 0 \\ 1 & 4 & 1 \\ 0 & 1 & 2\end{array}\right]\left[\begin{array}{c}u_{i-1}^{\bullet} \\ u_{i}^{\bullet} \\ u_{i+1}^{\bullet}\end{array}\right]$

(15) $=R \frac{l^{(e)}}{2}\left[\begin{array}{l}1 \\ 2 \\ 1\end{array}\right]$

Now, equate row corresponding to the node $i$ to zero, From Eq. (16a) the difference schemes is

$$
\begin{aligned}
& \frac{1}{l^{(e)^{2}}}\left[-u_{i-1}+2 u_{i}-u_{i+1}\right]-\frac{P}{2 l^{(e)}}\left[-u_{i-1}+u_{i+1}\right] \\
& +\frac{M^{2}}{6}\left[u_{i-1}+u_{i}+u_{i+1}\right]+\frac{1}{6}\left[\dot{\left.u_{i-1}+4 \dot{u}_{i}+\dot{u}_{i+1}\right]}\right]=R
\end{aligned}
$$


Applying Crank-Nicholson method to the above equation, then we get

$$
\begin{aligned}
& A_{1} u_{i-1}^{j+1}+A_{2} u_{i}^{j+1}+A_{3} u_{i+1}^{j+1}= \\
& A_{4} u_{i-1}^{j}+A_{5} u_{i}^{j}+A_{6} u_{i+1}^{j}+P^{*}
\end{aligned}
$$

Where

$$
\begin{aligned}
& A_{1}=2+M^{2} k-6 r+3 P h r, A_{2}=8+12 r+4 M^{2} k \\
& A_{3}=2+M^{2} k-6 r-3 P h r, A_{4}=2-M^{2} k+6 r-3 P h r \\
& A_{5}=8-12 r-4 M^{2} k, \quad A_{6}=2-M^{2} k+6 r+3 P h r \\
& P^{*}=12(G r) k \theta_{i}^{j}+12(G m) k \varphi_{i}^{j}
\end{aligned}
$$

Applying similar procedure to Eq. (11) and Eq. (12) then we gets

$$
\begin{aligned}
& B_{1} \theta_{i-1}^{j+1}+B_{2} \theta_{i}^{j+1}+B_{3} \theta_{i+1}^{j+1}= \\
& B_{4} \theta_{i-1}^{j}+B_{5} \theta_{i}^{j}+B_{6} \theta_{i+1}^{j}+P^{* *} \\
& C_{1} \varphi_{i-1}^{j+1}+C_{2} \varphi_{i}^{j+1}+C_{3} \varphi_{i+1}^{j+1}= \\
& C_{4} \varphi_{i-1}^{j}+C_{5} \varphi_{i}^{j}+C_{6} \varphi_{i+1}^{j}
\end{aligned}
$$

Where

$$
\begin{array}{ll}
B_{1}=2 P_{1}-6 r+3 P P_{1} r h, & B_{2}=8 P_{1}+12 r \\
B_{3}=2 P_{1}-6 r-3 P P_{1} r h, & B_{4}=2 P_{1}+6 r-3 P P_{1} r h \\
B_{5}=8 P_{1}-12 r, & B_{6}=2 P_{1}+6 r+3 P P_{1} r h \\
P^{* *}=12 P_{1} r E c(u[i+1]-u[i])^{2} \\
C_{1}=2 S c-6 r+3 P S c r h+Q k, & C_{2}=8 S c+12 r+4 Q k \\
C_{3}=2 S c-6 r-3 P S c & r h+Q k, C_{4}=2 S c+6 r-3 P S c r h-Q k \\
C_{5}=8 S c-12 r-4 Q k, & C_{6}=2 S c+6 r+3 P S c r h-Q k
\end{array}
$$

Here $Q=S c K_{r}^{2}, P_{1}=\frac{\operatorname{Pr}}{1+R}, r=\frac{k}{h^{2}}$ and $h, k$ are the mesh sizes along $y$-direction and time $t$-direction respectively. Index $i$ refers to the space and $j$ refers to the time. In Eqs. (17)-(19), taking $\mathrm{i}=1(1) \mathrm{n}$ and using initial and boundary conditions (Eq. (14)), the following system of equations in matrix notation are obtained:

$$
A_{i} X_{i}=B_{i} \quad i=1(1) 3
$$

Where $A_{i}$ 's are matrices of order $n$ and $X_{i}, B_{i}$ 's column matrices having $n$-components. The solutions of above system of equations are obtained by using Thomas algorithm for velocity, temperature and concentration. Also, numerical results for these equations are obtained by C-program. In order to prove the convergence and stability of Galerkin finite element method, the same C-program was run with slightly changed values of $h$ and $k$ and no significant change was observed in the values of $u, \theta$ and $\varphi$. Hence, the Galerkin finite element method is stable and convergent.

The skin-friction, Nusselt number and Sherwood number are important physical parameters for this type of boundary layer flow.
The skin-friction at the plate, which in the nondimensional form is given by

$$
C_{f}=\frac{\tau_{w}^{\prime}}{\rho U_{0} V_{0}}=\left(\frac{\partial u}{\partial y}\right)_{y=0}
$$

The rate of heat transfer coefficient, which in the nondimensional form in terms of the Nusselt number is given by

$N u=-x \frac{\left(\frac{\partial T}{\partial y^{\prime}}\right)_{y^{\prime}=0}}{T_{w}-T_{\infty}} \Rightarrow N u \operatorname{Re}_{x}^{-1}=-\left(\frac{\partial \theta}{\partial y}\right)_{y=0}$

The rate of heat transfer coefficient, which in the nondimensional form in terms of the Sherwood number, is given by

$S h=-x \frac{\left(\frac{\partial C}{\partial y^{\prime}}\right)_{y^{\prime}=0}}{C_{w}-C_{\infty}} \Rightarrow S h \operatorname{Re}_{x}^{-1}=-\left(\frac{\partial \varphi}{\partial y}\right)_{y=0}$

Where $\operatorname{Re}_{x}=\frac{V_{0} x}{v}$ is the local Reynolds number.

\section{Result AND Discussion}

In the preceding sections, the problem of an unsteady free convective flow of a viscous, incompressible, radiating and dissipating fluid past a semi-infinite plate with chemically reacting was formulated and solved by finite element technique. The expressions for the velocity, temperature and concentration were obtained. To illustrate the behavior of these physical quantities, numeric values were computed with respect to the variations in the governing parameters viz., the thermal Grashof number $\mathrm{Gr}$, Modified Grashof number $\mathrm{Gm}$, Eckert number $E c$, radiation parameter $R$, Prandtl number Pr, Schmidt number Sc and chemical reaction parameter $k_{r}$.

The temperature and the species concentration are coupled to the velocity via Grashof number $\mathrm{Gr}$ and Modified Grashof number Gm as seen in Eq. (11). For various values of Grashof number, the velocity profiles $u$ are plotted in Fig. (1) and Fig. (2). The Grashof number $G r$ signifies the relative effect of the thermal buoyancy force to the viscous hydrodynamic force in the boundary layer. As expected, it is observed that there is a rise in the velocity due to the enhancement of thermal buoyancy force. Also, as $G r$ increases, the peak values of the velocity increases rapidly near the porous plate and then decays smoothly to the free stream velocity. The Modified Grashof number $G m$ defines the ratio of the species buoyancy force to the viscous hydrodynamic force. As expected, the fluid velocity increases and the peak value is more distinctive due to increase in the species buoyancy force. The velocity distribution attains a distinctive maximum value in the vicinity of the plate and then decreases properly to approach the free stream value. It is noticed 
that the velocity increases with increasing values of Modified Grashof number (Gm).

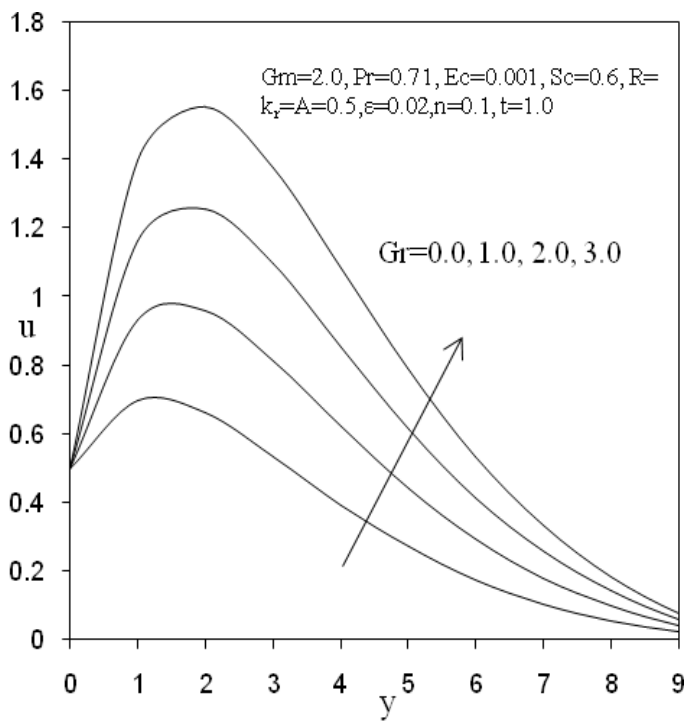

Fig. 1. Velocity profiles for different values of $\mathrm{Gr}$

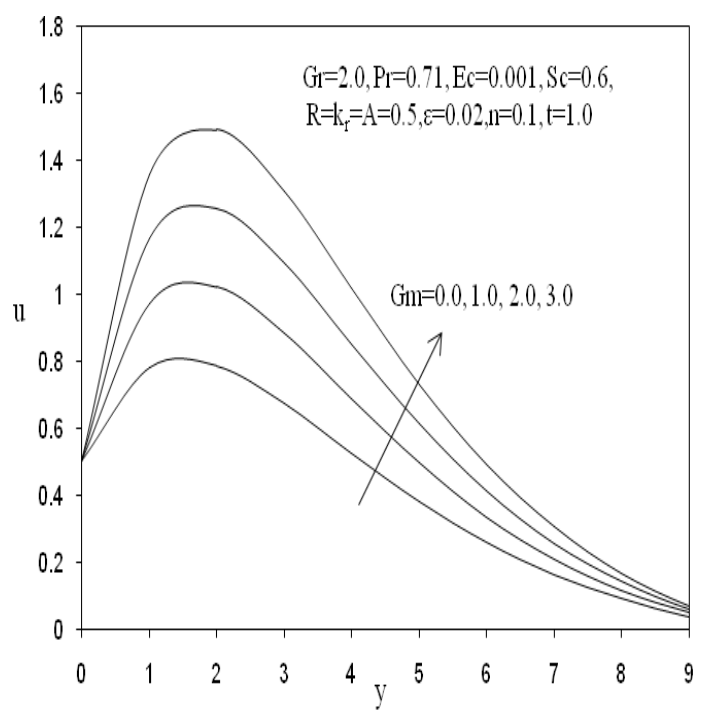

Fig. 2. Velocity profiles for different values of Gm

The Grashof number $G r$ signifies the relative effect of the thermal buoyancy force to the viscous hydrodynamic force

Fig. 3(a) and Fig. 3(b) show the velocity and temperature profiles for different values of the Radiation parameter $R$, it can be seen that an increase in the thermal radiation parameter produces significant increases in the thermal condition of the fluid and its thermal boundary layer. Through the buoyancy effect this increase in the fluid temperature induces more flow in the boundary layer causing the velocity of fluid there to increase. In addition the hydrodynamic boundary layer thickness increases as a result of increasing $R$. Further it is observed that the time required to reach the steady state increases with the increase in $R$.

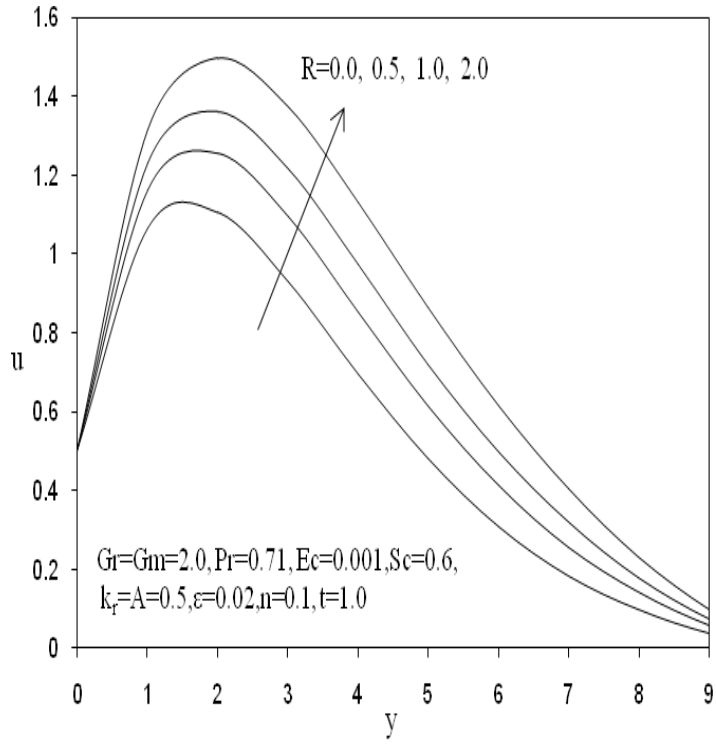

Fig. 3(a). Velocity profiles for different values of $R$

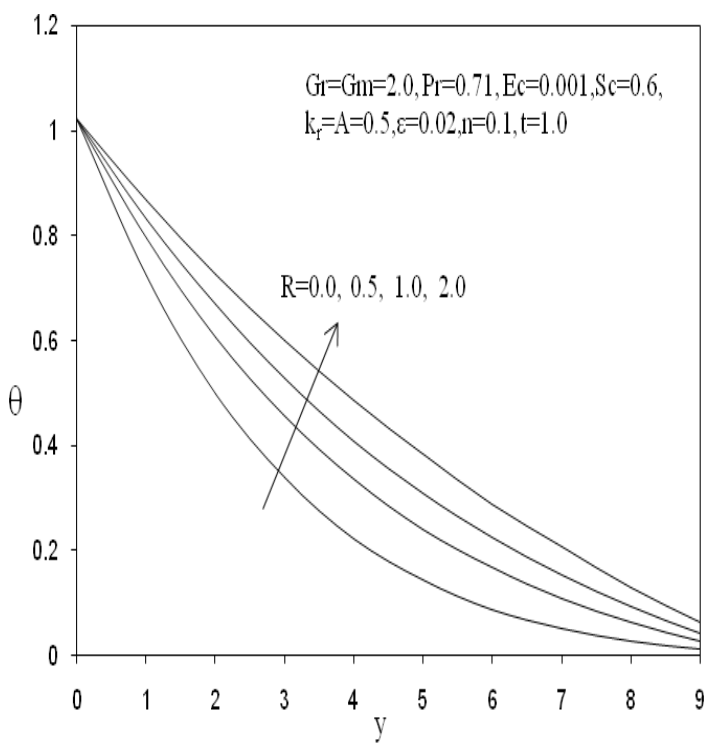

Fig. 3(b). Temperature profiles for different values of $R$

The effects of the viscous dissipation parameter i.e., Eckert number on the velocity and temperature are shown in Fig.4 (a) and Fig.4 (b) respectively. The Eckert number (Ec) expresses the relationship between the kinetic energy in the flow and the enthalpy. It embodies the conversion of kinetic energy into internal energy by work done against the viscous fluid stresses. Greater viscous dissipative heat causes a rise in the temperature as well as the velocity. This behavior is evident from Fig. 4(a) and Fig.4 (b). 


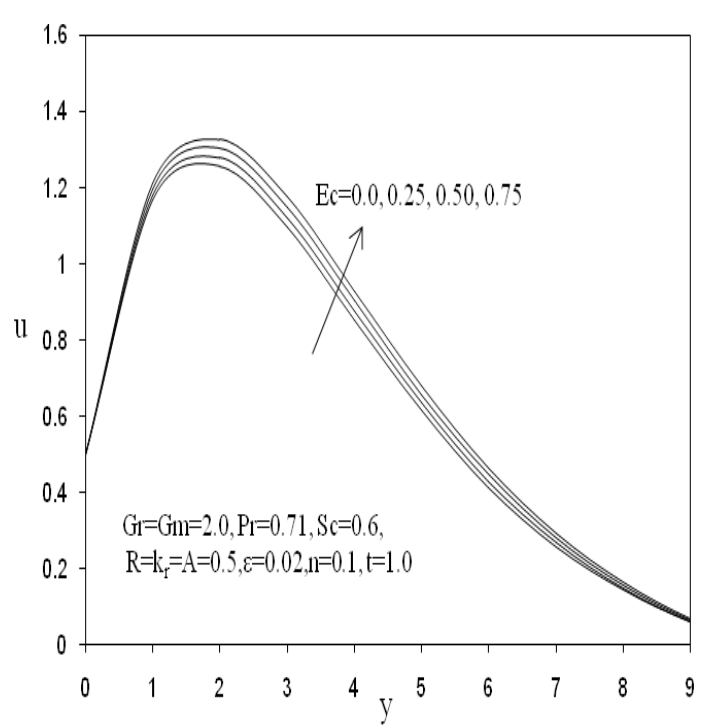

Fig. 4(a). Velocity profiles for different values of Ec

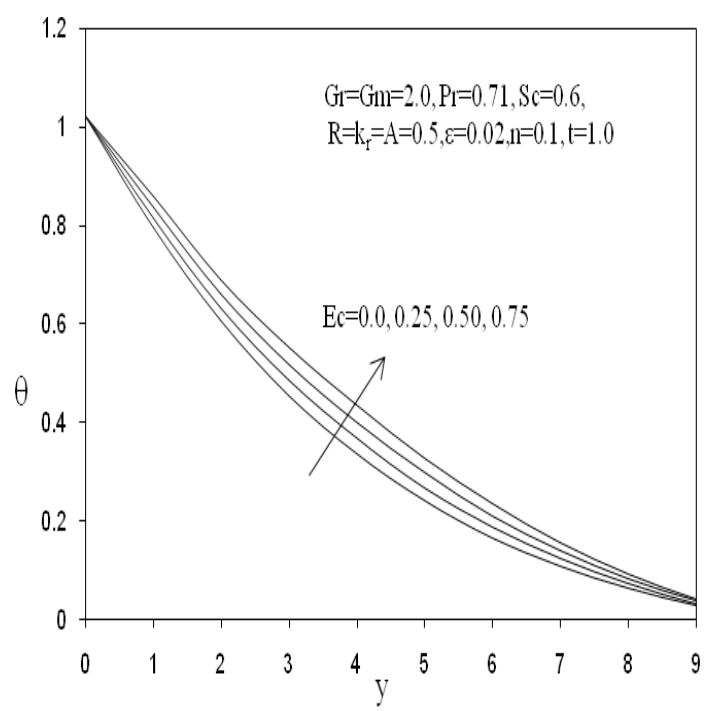

Fig. 4(b). Temperature profiles for different values of $\mathrm{Ec}$

The effect of the Prandtl number on the velocity and temperature are shown in Fig 5(a) and Fig 5(b). As the Prandtl number increases, the velocity and temperature decreases. The values of the Prandtl number are chosen for Mercury $(\operatorname{Pr}=0.025)$, air $(\operatorname{Pr}=0.71)$, electrolytic solution $(\mathrm{Pr}=1.0)$, water $(\mathrm{Pr}=7.0)$ and water at $4 \mathrm{oC}$ $(\mathrm{Pr}=11.40)$. In Fig 5(b) we depict the effect of Prandtl number (Pr) on the temperature field. It is observed that an increase in the Prandtl number leads to decrease in the temperature field. Also, temperature field falls more rapidly for water in comparison to air and the temperature curve is exactly linear for mercury, which is more sensible towards change in temperature. From this observation, it is concluded that mercury is most effective for maintaining temperature differences and can be used efficiently in the laboratory. Air can replace mercury, the effectiveness of maintaining temperature changes are much less than mercury. However, air can be better and cheap replacement for industrial purpose. This is because, either increase of kinematic viscosity or decrease of thermal conductivity leads to increase in the value of Prandtl number (Pr). Hence temperature decreases with increasing of Prandtl number (Pr).

For various values of the magnetic parameter $\mathrm{M}$ the velocity profiles are plotted in Fig 5(c). The influence of the magnetic parameter $\mathrm{M}$ on the velocity profiles is an expected. As $M$ increase the Lorentz force which oppose the flow also increases and leads to enhanced deceleration of the flow this result qualitatively agrees with the expectations, since the magnetic field exact a retarding force on the free convective flow the obvious from the decrease in the velocity profiles in Fig 5(c).

The effect of the Schmidt number on the velocity and concentration are shown in Fig 6(a) and Fig 6(b). To focus out attention on numerical values of the results obtained in the study the values of Sc are chosen for the gases representing diffusing chemical species of most common interest in air namely Hydrogen $(\mathrm{Sc}=0.22)$, Water-vapour $(\mathrm{Sc}=0.60)$, Oxygen $(\mathrm{Sc}=0.66)$, Ammonia $(\mathrm{Sc}=0.78)$, Methanol $(\mathrm{Sc}=1.00)$ and Propyl-benzene $(\mathrm{Sc}=2.62)$ at $20 \mathrm{oC}$ and one atmospheric pressure. As the Schmidt number increases, the velocity and concentration decreases. This causes the concentration buoyancy effects to decrease yielding a reduction in the fluid velocity. Reductions in the velocity and concentration distributions are accompanied by simultaneous reductions in the velocity and concentration boundary layers.

Fig. 6(b) shows the concentration field due to variation in Schmidt number $(S c)$ for the gasses Hydrogen, Water - vapour, Oxygen, Ammonia and Methanol. It is observed that concentration field is steady for Hydrogen and falls rapidly for Oxygen and Ammonia in comparison to Water - vapour. Thus, Hydrogen can be used for maintaining effective concentration field and Water - vapour can be used for maintaining normal concentration field.

Fig. 7(a) and Fig.7 (b) illustrate the behavior of velocity and concentration for different values of chemical reaction parameter $k_{r}$. It is observed that an increase in chemical reaction parameter leads to a decrease in both the values of velocity and concentration.

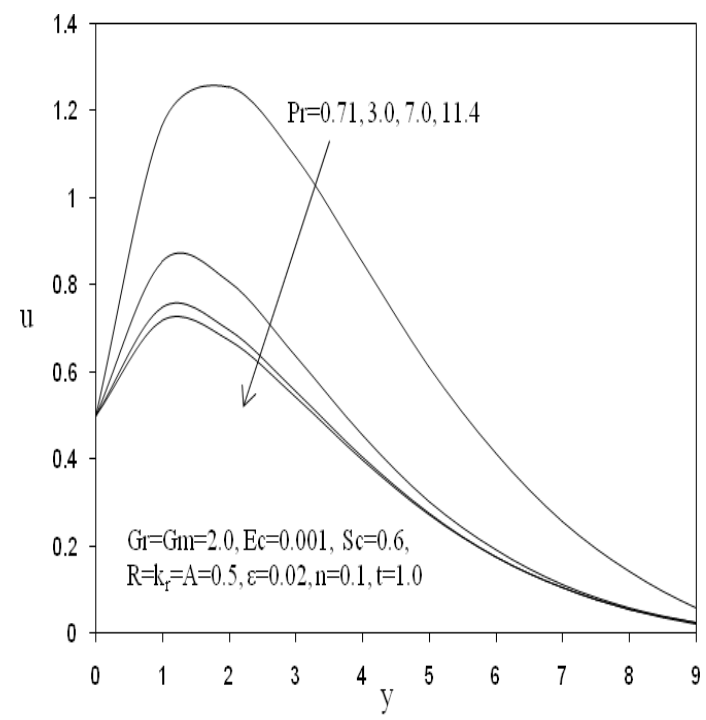

Fig. 5(a). Velocity profiles for different values of $\mathrm{Pr}$ 


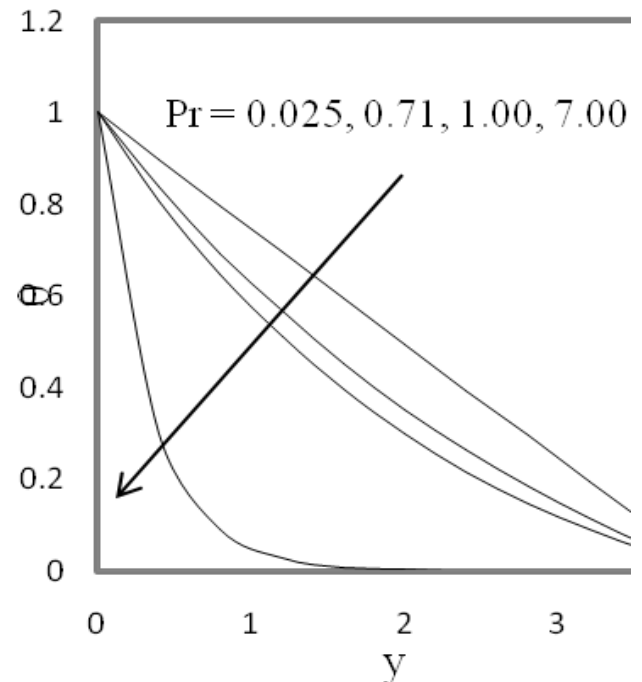

Fig. 5(b). Temperature profiles for different values of $\operatorname{Pr}$

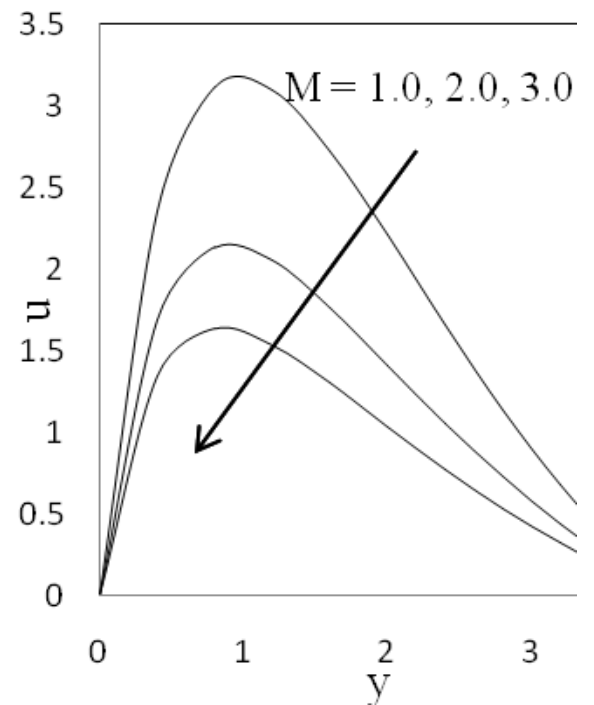

Fig. 5(c). Velocity profiles for different values of M

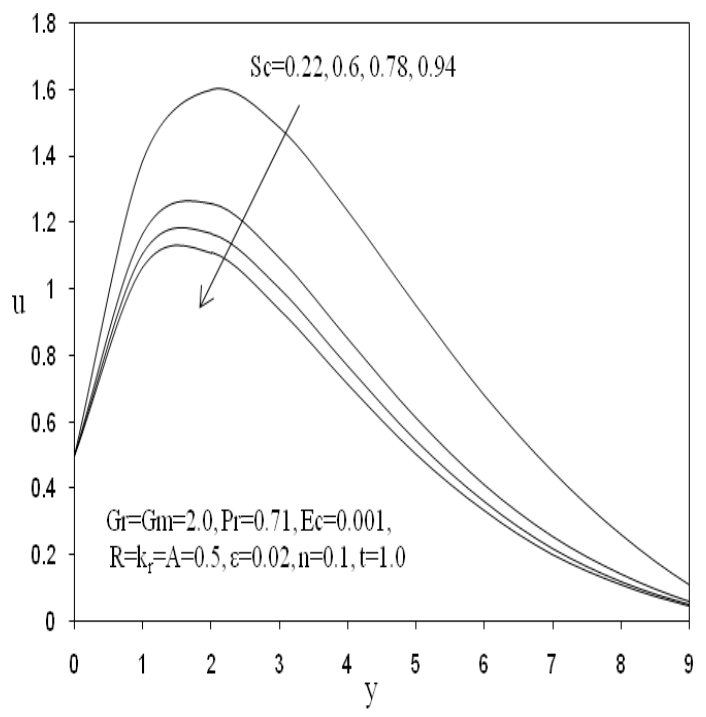

Fig. 6(a). Velocity profiles for different values of Sc

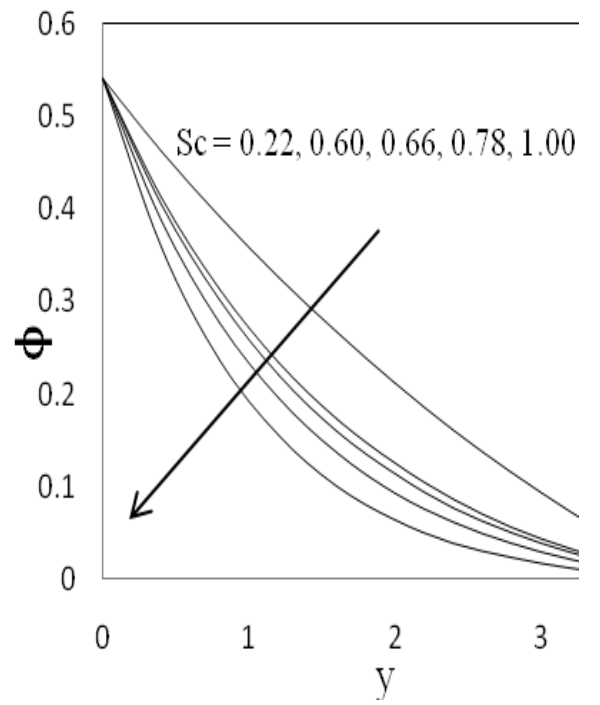

Fig. 6(b). Concentration profile for different values of $\mathrm{Sc}$

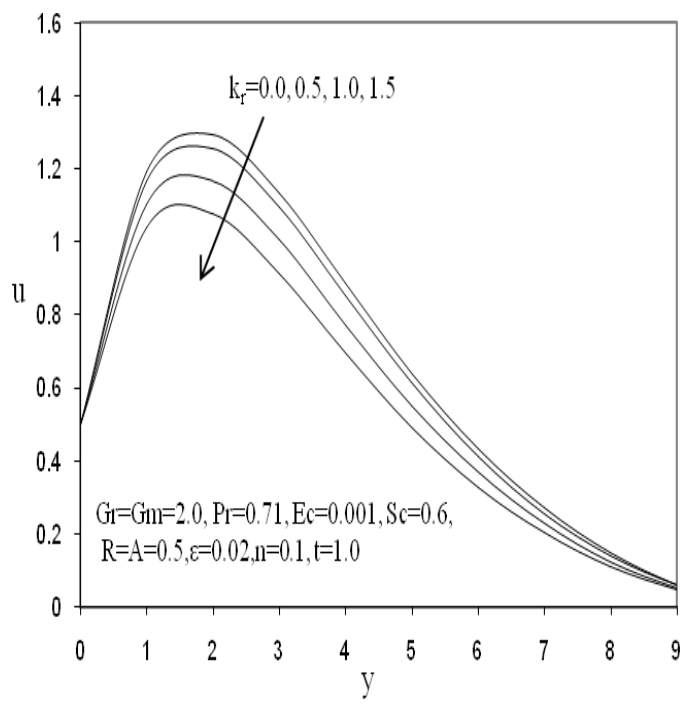

Fig. 7(a). Velocity profiles for different values of $\mathrm{Kr}$

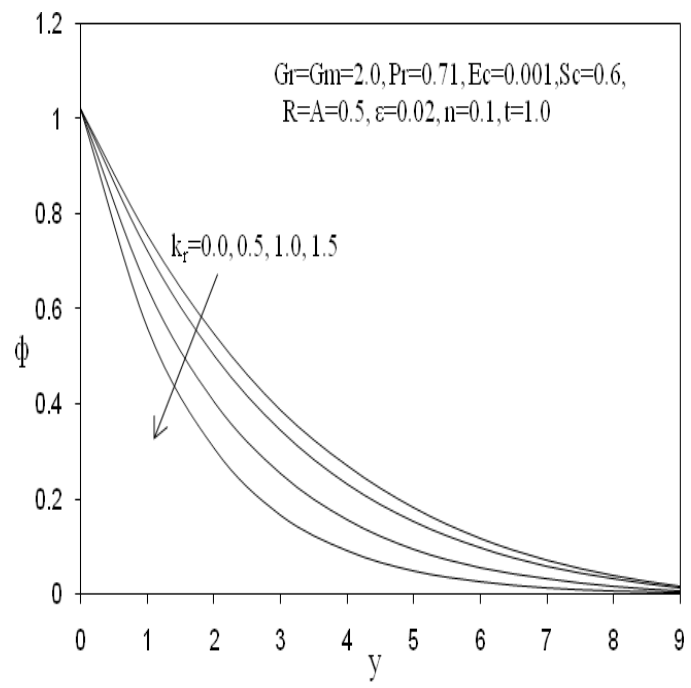

Fig. 7(b). Concentration profiles for different values of $\mathrm{Kr}$ 
Table 1-5 present the effects of the thermal Grashof number, Modified Grashof number, Radiation parameter, Schmidt number and Eckert number on the skin-frication coefficient, Nusselt number and Sherwood number. From Table 1 and Table 2, it is observed that as $G r$ or $G m$ increases, the skin friction coefficient increases. However, from Table 3, it can be seen that as the radiation parameter increases, the skin-friction coefficient increases and Nusselt number decreases. From Table 4, it is noticed that an increase in the Schmidt number reduces the skinfriction coefficient and increases the Sherwood number. Finally, it is observed from Table 5 that as Eckert number increases, the skin-friction coefficient increases and the Nusselt number decreases.

Table 1 Effect of $G r$ on $C_{f}$ Reference values as in Fig.1.

\begin{tabular}{|c|c|}
\hline$G r$ & $C_{f}$ \\
\hline 0.0 & 0.8343 \\
1.0 & 1.6445 \\
2.0 & 2.4548 \\
3.0 & 3.2652 \\
\hline
\end{tabular}

Table 2 Effect of $G m$ on $C_{f}$ Reference values as in Fig.1.

\begin{tabular}{|c|c|}
\hline$G m$ & $C_{f}$ \\
\hline 0.0 & 1.0816 \\
1.0 & 1.7682 \\
2.0 & 2.4548 \\
3.0 & 3.1414 \\
\hline
\end{tabular}

Table 3 Effect of $R$ on $C_{f}$ and $N u$ Reference values as in Fig.3 (a)

\begin{tabular}{|c|c|c|}
\hline$R$ & $C_{f}$ & $N u$ \\
\hline 0.0 & 2.1664 & 0.8365 \\
0.5 & 2.4548 & 0.6139 \\
1.0 & 2.6536 & 0.5032 \\
2.0 & 2.9037 & 0.4010 \\
\hline
\end{tabular}

Table 4 Effect of $S c$ on $C_{f}$ and $S h$ Reference values as in Fig.3 (a)

\begin{tabular}{|c|c|c|}
\hline$S c$ & $C_{f}$ & $S h$ \\
\hline 0.22 & 3.1068 & 0.4515 \\
0.60 & 2.4548 & 0.8431 \\
0.78 & 2.2767 & 1.0214 \\
0.94 & 2.1540 & 1.1745 \\
\hline
\end{tabular}

Table 5 Effect of Ec on $\mathrm{Cf}$ and $\mathrm{Nu}$ Reference values as in Fig.3 (a)

\begin{tabular}{|c|c|c|}
\hline Ec & Cf & $\mathrm{Nu}$ \\
\hline 0.0 & 2.4546 & 0.6143 \\
0.25 & 2.5010 & 0.5130 \\
0.50 & 2.5489 & 0.4039 \\
0.75 & 2.5985 & 0.2863 \\
\hline
\end{tabular}

\section{Conclusions}

We have formulated the problem of two-dimensional fluid flow in the presence of radiative heat transfer, viscous dissipation and chemical reaction. A finite element technique is employed to solve the resulting coupled partial differential equations. The following conclusions are drawn from the study.

1. The velocity increases with the increase in thermal Grashof number and Modified Grashof number.

2. An increase in the Eckert number increases the velocity and temperature.

3. An increase in the Prandtl number decreases the velocity and temperature.

4. An increase in the radiation parameter leads to increase in the velocity and temperature.

5. The velocity as well as concentration decreases with an increase in the Schmidt number.

6. The velocity as well as concentration decreases with an increase in the chemical reaction parameter.

\section{REFERENCES}

Anjali Devi, S.P., and B.Ganga (2009). Dissipation effects on MHD Non linear flow and heat transfer past a porous surface with prescribed heat flux. Journal of Applied Fluid Mechanics Vol 3(1), 1-6.

Chamkha, A.J., H.S. Takhar and V.M.Soundalgekar (2001). Radiation Effects on free convection flow past a Semi-infinite vertical Plate with Mass Transfer. Chemical Engineering Journal 84, 335342.

Das,U.N., R.K.Deka and V.M.Soundalgekar (1994). Effects of Mass Transfer on Flow Past an Impulsively started Infinite Vertical Plate with Constant Heat Flux and Chemical reaction. Forschung in Inge-Engg Reseach 60, 284-287.

Gebhar, B. (1962). Effects of viscous dissipative in natural convection. Journal of Fluid Mech 14, 225232. 
V.S. Rao et al. / JAFM, Vol. 6, No. 3, pp. 321-329, 2013.

Hossain, M.A., and H.S.Takhar, (1996). Radiation Effects on mixed convection along a vertical plate with Uniform Surface Temperature. Heat and Mass Transfer 31, 243-248.

Israel-Cookey, C., A.Ogulu and V.B.Ombo Pepple (2003). Viscous dissipation and radiation on unsteady MHD free convection flow past an infinite heated vertical plate in porous medium with time dependent suction. Int J Heat Mass Transfer 46, 2305 .

Kim,Y.J., and A.G.Fedorov (2003) Transient Mixed Radiative convection flow of a micro polar fluid past a moving, semi-infinite vertical porous plate. International journal of Heat and Mass Transfer $46,1751-1758$.
Prakash, J., and A. Ogulu (2006). Unsteady TwoDimensional Flow of a Radiating and Chemically Reacting MHD Fluid with Time-dependent Suction. Indian J. Pure and Applied Physics 44, 805-810.

Raptis, A., and C.Perdikis (1999). Radiation and free convection flow past a moving plate. Appl.Mech.Eng. 4, 817-821.

Soundalgekar,V.M.(1972).Viscous Dissipative effects on unsteady free convective flow past an vertical porous plate with constant suction. Int. J. Heat Mass Transfer 15, 1253-1261.

Soundalgekar,V.M., and H.S.Takhar (1993). Radiation Effects on free convection flow past a semi-infinite vertical plate. Modeling Measurement and Control R 51, 31-40. 